\title{
Observations and modeling of the ultraviolet emission of solar flares
}

\author{
Katarzyna Mikuła ${ }^{1}$, Arkadiusz Berlicki ${ }^{1,2}$ and Petr Heinzel ${ }^{2}$ \\ ${ }^{1}$ Astronomical Institute, University of Wrocław, Poland \\ email: mikula@astro.uni.wroc.pl \\ email: berlicki@astro.uni.wroc.pl \\ ${ }^{2}$ Astronomical Institute, Czech Academy of Sciences, Czech Republic \\ email: petr.heinzel@asu.cas.cz
}

\begin{abstract}
In this paper we present the method of using far UV spectra of the flare observed by Interface Region Imaging Spectrograph (IRIS) for determination of the contribution of the continuum emission to the total UV radiation observed e.g. by SDO in $1600 \AA$ channel. In our method the Si IV (1402.77 $\AA$ ) line observed by IRIS is used as a proxy of C IV line emission contained in SDO/AIA UV images. Determined intensity of the flare continuum emission can be used to study the physics of the flare heated chromosphere and for better understanding of the emission mechanisms.
\end{abstract}

Keywords. Sun: chromosphere, Sun: flares, Sun: transition region, Sun: UV radiation

\section{Introduction}

The solar chromosphere and the solar transition region, heated during solar flares, strongly emits in several spectral lines in the ultraviolet range (UV) e.g. in Si IV, O IV and C IV. Very often the UV images from space telescopes are mixture of emission from different spectral lines and continuum. Studying resonance lines like Si IV (1393.76 A, $1402.77 \AA$ ) and C IV (1548.19 A, $1550.76 \AA$ ) we can separate contribution from UV spectral lines emission from the UV continuum. In this way we get pure UV continuum intensity of solar flares. Understanding of the continuum emission of flares is important for the analysis of their chromospheric heating mechanisms. In our analysis we used IRIS observations of solar flare which occurred on March 29, 2014. We study FUV spectra of this flare, in particular Si IV line (1402.77 $\AA$ ) and we attempt to model its ultraviolet emission.

\section{Flare observations and observed FUV spectrum}

On March 29, 2014, NOAA active region 12017 (part of a larger AR 12018), centered at $\left(\sim 10^{\circ} \mathrm{N}, 33^{\circ} \mathrm{W}\right)$, produced an X1.0 class solar flare that began at $\sim 17: 35 \mathrm{UT}$ with the peak reached at $\sim 17: 48 \mathrm{UT}$ (according to the X-ray flux recorded by GOES). This flare was also observed by IRIS in three spectral windows: near UV (NUV) and far UV (FUV1, FUV2). IRIS also obtained slit-jaw images (SJI) for three passbands: $1400 \AA$ (transition region), $2796 \AA$ (upper chromosphere) and $2832 \AA$ (photospheric image). During the flare observations (from 14:09 UT to 17:54 UT) IRIS performed 180 rasters with 8 slit positions and the steps of $2^{\prime \prime}$. These parameters give the total field-of-view of $14^{\prime \prime} \times 174^{\prime \prime}$ for spectra and $174^{\prime \prime} \times 174^{\prime \prime}$ for SJIs.

We focus on the spectra from both FUV channels, which contain five spectral regions: $\sim 1333$ - $1337 \AA$ (C II lines), 1342 - 1344 A (continuum), 1348 - $1351 \AA$ (Fe XII line), 

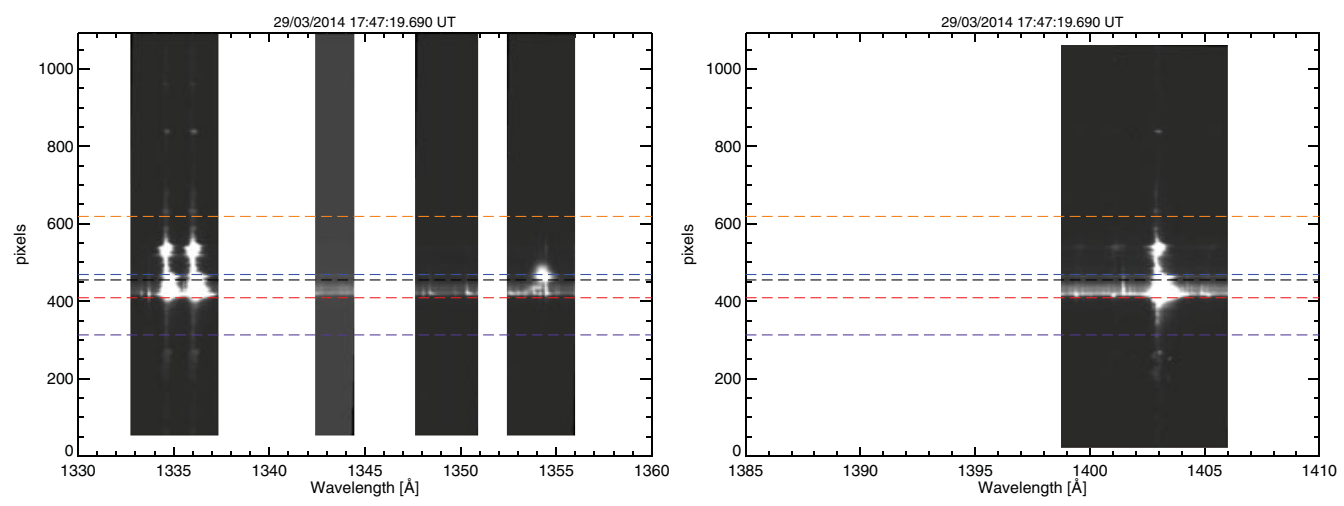

Figure 1. IRIS FUV spectrum showing the flare intensity increase throughout the spectrum (C II, $1343 \AA$, Fe XII, O I and Si IV lines), including the continuum. The color lines refer to the positions along the slit used for the analysis: purple: $\mathrm{x}=314$, red: $\mathrm{x}=423$, black: $\mathrm{x}=447$, blue: $\mathrm{x}=468$ and orange: $\mathrm{x}=620$.

$\sim 1353$ - $1356 \AA$ (Fe XXI and O I lines) and $\sim 1399$ - $1406 \AA$ (Si IV line). In the analysis we used calibrated data (IRIS Level 2 data), which are corrected for dark current, flat-field, geometry and wavelength calibration but without radiometric calibration. We chose the FUV spectrum obtained at 17:47:19.690 UT at five positions along the slit (Fig. 1). Due to the intensity saturation in strong UV lines (C II and Si IV) we couldn't choose the positions corresponding to the most intense parts of the flare spectral lines. Therefore, we took nearby positions, close to the flare brightenings and two positions outside the flare ( $\mathrm{y}=314$ and $\mathrm{y}=620$, cospatial with the positions used in Heinzel \& Kleint (2014)). Intensities in IRIS data are given in Data Number (DN) or counts (DN s ${ }^{-1}$ ). We had to perform radiometric calibration of the observed spectra to obtain the intensities in absolute units $\left[\mathrm{erg} \mathrm{s}{ }^{-1} \mathrm{~cm}^{-2} \mathrm{sr}^{-1} \AA^{-1}\right]$.

\section{Modeling of the UV emission of the solar flare and preliminary results}

For the modeling of the ultraviolet emission of the flare we used CHIANTI database. In CHIANTI software it is necessary to provide the differential emission measure (DEM) and chemical abundances. At first, we calculated DEMs based on semiempirical flare model atmospheres F1 and F2 (Machado et al. 1980). Then we used abundances of silicon from Sylwester et al. (2015), determined from RESIK crystal spectrometer data. The silicon abundances are $\mathrm{A}(\mathrm{Si})=7.53 \pm 0.08$. Carbon abundances $(\mathrm{A}(\mathrm{C})=8.59)$ were taken from Feldman et al. (1992). For this conditions we calculated synthetic UV spectra in ranges corresponding to both IRIS FUV channels. CHIANTI database takes into account Maxwellian distribution of the electrons. Nevertheless, non-Maxwellian distribution of the electrons (called $\kappa$-distribution, Dzifčáková et al. 2015) has been detected in the transition region during solar flares. Therefore, we used KAPPA package for our calculations of the synthetic spectra.

Here, we focus on the spectra obtained for $\mathrm{y}=456$ position for C II and Si IV lines. Fig. 2 shows fitted synthetic spectral lines for $\mathrm{y}=456$ position. Black lines are observational line profiles. In this case, the best fitting was found for $\mathrm{F} 2$ model, $\kappa=4$, and FWHM $=$ 0.30 (Si IV line) and FWHM = 0.35 (C II line). Based on these conditions we calculated synthetic spectra for C IV lines (1548.19 A and $1550.76 \AA$ ). Bottom panel (Fig. 2) shows 

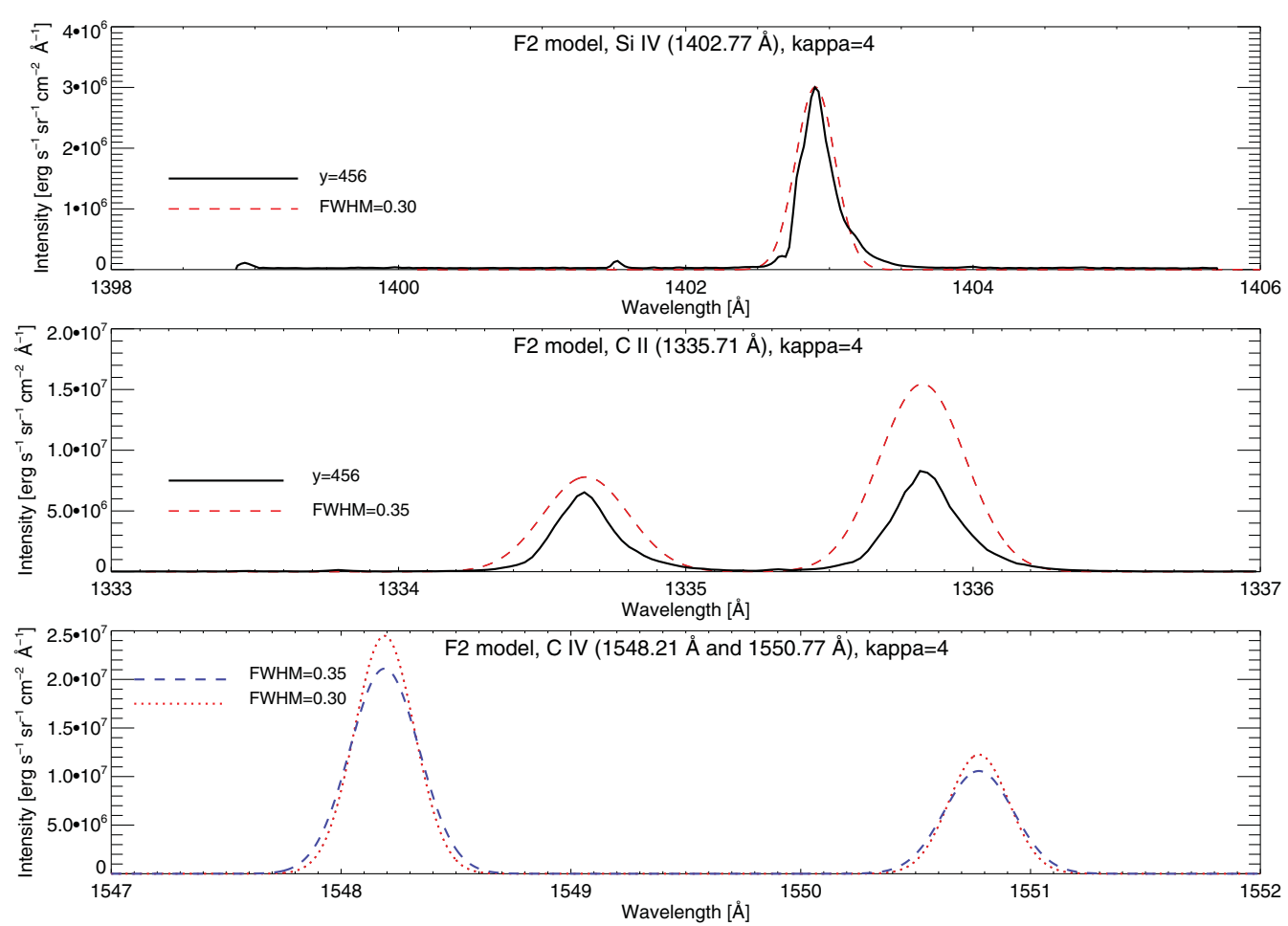

Figure 2. The observed SiIV and CII line profiles for $y=410$ (upper and middle panel black lines), fitted with the synthetic spectra obtained with KAPPA package for F2 model for $\kappa=4$ (red dashed lines). Lower panel shows synthetic C IV line profiles calculated with KAPPA package for the same conditions and for two FWHM values (red and blue lines).

C IV theoretical line profiles calculated for F2 model, $\kappa=4$ and for two values of FWHM $=0.35$ and 0.30 .

In the next step we will calibrate the images from SDO/AIA in $1600 \AA$ channel to get the intensities in physical units. Knowing the CIV line emission obtained from the modeling of IRIS spectra we can subtract it from AIA images and got pure continuum emission for the whole flare area.

\section{Acknowledgement}

This project has received funding from the European Communitys Seventh Framework Programme (FP7/2007-2013) under grant agreement no. 606862 (F-CHROMA). This work is also supported by the institutional project RVO: 67985815 of the Astronomical Institute of the Academy of Sciences of the Czech Republic (AB, PH).

\section{References}

Dzifčáková, E., Dudík, J., Kotrč, P., Fárník, F., \& Zemanová, A. 2015, ApJS, 217, 14

Feldman, U., Mandelbaum, P., Seely, J. L., \& Doschek, G. A., Gursky H. 1992, ApJSS, 81, 387 Heinzel, P. \& Kleint, L. 2014, ApJ, 794, 23

Machado, M. E., Avrett, E. H., Vernazza, J. E., \& Noyes, R. W. 1980, ApJ, 242, 336

Sylwester, B., Phillips, K. J. H., Sylwester, J., \& Kȩpa, A. 2015, ApJ, 805, 49 